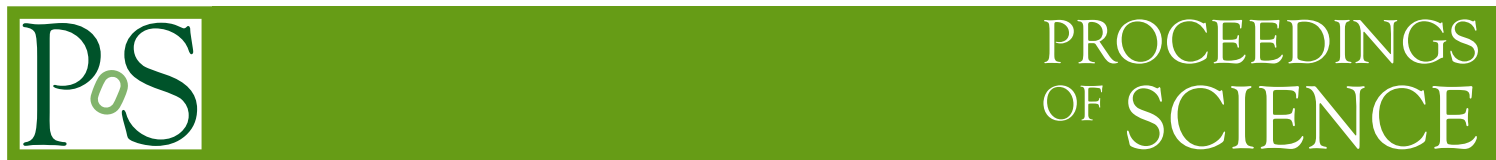

\title{
Momentum Dependent Two-Loop Corrections to the Neutral Higgs Boson Masses in the MSSM
}

\author{
Sophia Borowka* \\ Max Planck Institute for Physics, Föhringer Ring 6, 80805 Munich, Germany \\ E-mail: sborowka@mpp.mpg.de
}

\begin{abstract}
The momentum dependent two-loop contributions of the order $\mathscr{O}\left(\alpha_{t} \alpha_{s}\right)$ to the masses in the Higgs-boson sector of the MSSM are computed. Adopting the Feynman-diagrammatic approach

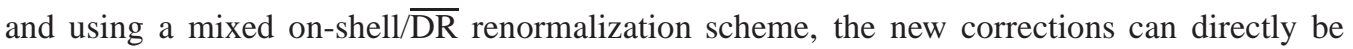
matched onto the higher-order corrections included in the code FEYNHIGGS. Two-loop diagrams involving several mass scales are evaluated with the program SECDEC. The combination of the new momentum dependent two-loop contribution with the existing one- and two-loop corrections leads to an improved prediction of the light MSSM Higgs-boson mass with reduced theoretical uncertainty. The resulting shifts in the lightest Higgs-boson mass $M_{h}$ can extend up to the level of the current experimental uncertainty of about $500 \mathrm{MeV}$ in the scenario considered in these proceedings.
\end{abstract}

Loops and Legs in Quantum Field Theory - LL 2014,

27 April - 2 May 2014

Weimar, Germany

\footnotetext{
*Speaker.
} 


\section{Introduction}

The ATLAS and CMS experiments at CERN have discovered a new boson with a mass around 125.6 GeV [1,2]. Despite its seemingly Standard Model-like behavior within the present experimental uncertainties, the newly discovered particle can also be interpreted as the Higgs-boson of extended models. The Higgs-boson sector of the Minimal Supersymmetric Standard Model (MSSM) [3] with two scalar doublets accommodates five physical Higgs-bosons, the light and heavy $C P$-even bosons $h^{0}$ and $H^{0}$, the $C P$-odd boson $A^{0}$, and the charged Higgs-bosons $H^{ \pm}$. The light $C P$-even Higgs-boson $h^{0}$ can be identified with the newly discovered scalar particle. Scenarios where the latter is associated with the heavy $C P$-even Higgs-boson $H^{0}$ is not considered in these proceedings. In the MSSM, the mass of $h^{0}, M_{h}$, can directly be predicted from the other parameters of the model. The accuracy of this prediction should at least match the one of the experimentally measured mass value for the new boson.

The status of higher-order corrections to the masses and mixing angles in the neutral Higgsboson sector of the MSSM with real parameters is quite advanced. The complete one-loop result within the MSSM is known [4-7]. The dominant one-loop contributions are the ones of order $\alpha_{t}$ originating from top and stop loops $\left(\alpha_{t} \equiv y_{t}^{2} /(4 \pi)\right.$ and $y_{t}$ being the top-quark Yukawa coupling). The range of available two-loop corrections meanwhile also covers most of the contributions which are believed to be significant [8-22]. In particular, the $\mathscr{O}\left(\alpha_{t} \alpha_{s}\right)$ contributions to the self-energies evaluated in the Feynman-diagrammatic (FD) as well as in the effective potential (EP) approach as well as the $\mathscr{O}\left(\alpha_{t}^{2}\right), \mathscr{O}\left(\alpha_{b} \alpha_{s}\right), \mathscr{O}\left(\alpha_{t} \alpha_{b}\right)$ and $\mathscr{O}\left(\alpha_{b}^{2}\right)$ contributions - evaluated in the EP approach - are known for vanishing external momenta. The obtained results are publicly available in the code FeynHiggs [9, 23, 27-29].

An evaluation of the momentum dependence at the two-loop level in a calculation employing the $\overline{\mathrm{DR}}$ scheme was presented in Ref. [24]. A (nearly) full two-loop EP calculation, including even the leading three-loop corrections, has also been published [25]. However, within the EP method all contributions are evaluated at zero external momentum for the corresponding self-energies, in contrast to the FD method, which in principle allows non-vanishing external momentum. Further, the calculation presented in Ref. [25] is not publicly available as a computer code for Higgs-boson mass calculations. Subsequently, another leading three-loop calculation of $\mathscr{O}\left(\alpha_{t} \alpha_{s}^{2}\right)$ has been performed [26], using assumptions on the various SUSY mass hierarchies, resulting in the code $\mathrm{H} 3 \mathrm{M}$ (which adds the three-loop corrections to the FEYNHIGGS result). Most recently, a combination of the full one-loop result, supplemented with leading and sub-leading two-loop corrections evaluated in the Feynman-diagrammatic/effective potential approach and a resummation of the leading and sub-leading logarithmic contributions from the scalar-top sector has been published [27] and included in the latest version of the code FEYNHIGGS [9, 23, 27-29].

In these proceedings, the calculation of mass shifts resulting from the inclusion of the leading momentum-dependent $\mathscr{O}\left(\alpha_{t} \alpha_{s}\right)$ corrections to the neutral $C P$-even Higgs-boson masses is described for one representative scenario. Further scenarios and more details are found in Ref. [30]. 


\section{Outline of the calculation}

The MSSM requires two doublets $\mathscr{H}_{1}$ and $\mathscr{H}_{2}$ of complex scalar fields which read

$$
\mathscr{H}_{1}=\left(\begin{array}{c}
\mathscr{H}_{1}^{0} \\
\mathscr{H}_{1}^{-}
\end{array}\right)=\left(\begin{array}{c}
v_{1}+\frac{1}{\sqrt{2}}\left(\phi_{1}^{0}-i \chi_{1}^{0}\right) \\
-\phi_{1}^{-}
\end{array}\right), \mathscr{H}_{2}=\left(\begin{array}{c}
\mathscr{H}_{2}^{+} \\
\mathscr{H}_{2}^{0}
\end{array}\right)=\left(\begin{array}{c}
\phi_{2}^{+} \\
v_{2}+\frac{1}{\sqrt{2}}\left(\phi_{2}^{0}+i \chi_{2}^{0}\right)
\end{array}\right) .
$$

The vacuum expectation values $v_{1}$ and $v_{2}$ define the angle $\tan \beta=v_{2} / v_{1}$. At tree level, the mass matrix of the neutral CP-even Higgs-bosons in the $\left(\phi_{1}^{0}, \phi_{2}^{0}\right)$ basis can be written as

$$
M_{\mathrm{Higgs}}^{2, \text { tree }}=\left(\begin{array}{cc}
m_{A^{0}}^{2} \sin ^{2} \beta+m_{Z}^{2} \cos ^{2} \beta & -\left(m_{A^{0}}^{2}+m_{Z}^{2}\right) \sin \beta \cos \beta \\
-\left(m_{A^{0}}^{2}+m_{Z}^{2}\right) \sin \beta \cos \beta & m_{A^{0}}^{2} \cos ^{2} \beta+m_{Z}^{2} \sin ^{2} \beta
\end{array}\right),
$$

where $m_{A^{0}}$ is the mass of the CP-odd neutral Higgs-boson $A^{0}$. The rotation to the basis formed by the mass eigenstates $H^{0}, h^{0}$ is given by

$$
\left(\begin{array}{c}
H^{0} \\
h^{0}
\end{array}\right)=\left(\begin{array}{cc}
\cos \alpha & \sin \alpha \\
-\sin \alpha & \cos \alpha
\end{array}\right)\left(\begin{array}{l}
\phi_{1}^{0} \\
\phi_{2}^{0}
\end{array}\right)
$$

\subsection{Computational set-up}

The higher-order corrected $C P$-even Higgs-boson masses in the MSSM are obtained from the corresponding propagators dressed by their self-energies. The inverse propagator matrix in the $\left(\phi_{1}^{0}, \phi_{2}^{0}\right)$ basis is given by

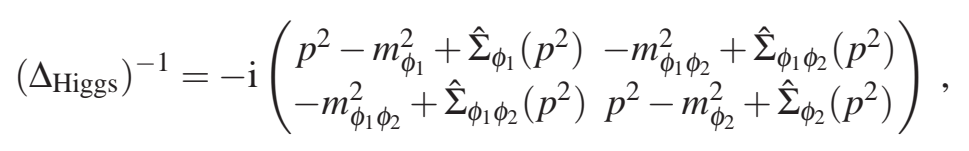

where the $\hat{\Sigma}\left(p^{2}\right)$ denote the renormalized Higgs-boson self-energies, $p$ being the external momentum.

The calculation is performed in the Feynman-diagrammatic (FD) approach. To obtain expressions for the unrenormalized self-energies at $\mathscr{O}\left(\alpha_{t} \alpha_{s}\right)$, the evaluation of genuine two-loop diagrams and one-loop graphs with counter-term insertions is required. Example diagrams for the neutral Higgs-boson self-energies are shown in Fig. 1. For the counter-term insertions, one-loop diagrams with external top quarks/squarks have to be evaluated. In addition, two-loop tadpole diagrams enter the two-loop counter terms. The complete set of contributing Feynman diagrams has been generated with the program FEYNARTS [31] (using the model file including counter terms from Ref. [32]). A tensor reduction and evaluation of traces was performed with the programs FORMCALC [33] and TwOCALC [34], yielding algebraic expressions in terms of the scalar one- and two-point one-loop functions, massive two-loop vacuum functions [35], and two-loop integrals which depend on the external momentum. The latter have been evaluated with the program SECDEC [36, 37].

\subsection{Computation of mass shifts}

The calculation of the self-energies is performed in the $\left(\phi_{1}^{0}, \phi_{2}^{0}\right)$ basis. To be consistent with the higher-order contributions to the Higgs-boson masses incorporated in the program FEYNHIGGS, 


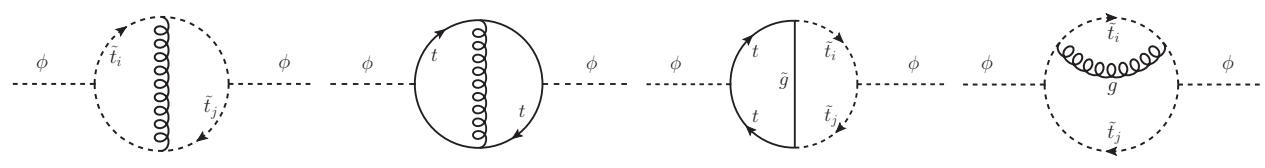

(a)

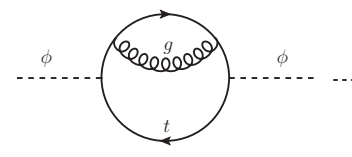

(e) (b)

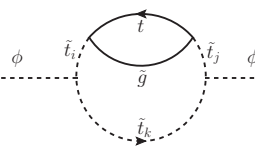

(f) (c)

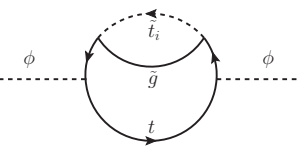

(g) (d)

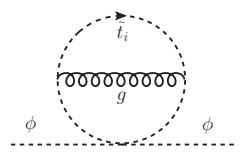

(h)

Figure 1: Examples of two-loop diagrams enetring the Higgs-boson self-energies $\left(\phi=h^{0}, H^{0}, A^{0}\right)$.

the renormalized self-energies in the $\left(\phi_{1}^{0}, \phi_{2}^{0}\right)$ basis are rotated into the physical $\left(h^{0}, H^{0}\right)$ basis,

$$
\begin{aligned}
& \hat{\Sigma}_{H^{0} H^{0}}^{(2)}=\cos ^{2} \alpha \hat{\Sigma}_{\phi_{1}^{0} \phi_{1}^{0}}^{(2)}+\sin ^{2} \alpha \hat{\Sigma}_{\phi_{2}^{0} \phi_{2}^{0}}^{(2)}+\sin (2 \alpha) \hat{\Sigma}_{\phi_{1}^{0} \phi_{2}^{0}}^{(2)}, \\
& \hat{\Sigma}_{h^{0} h^{0}}^{(2)}=\sin ^{2} \alpha \hat{\Sigma}_{\phi_{1}^{0} \phi_{1}^{0}}^{(2)}+\cos ^{2} \alpha \hat{\Sigma}_{\phi_{2}^{0} \phi_{2}^{0}}^{(2)}-\sin (2 \alpha) \hat{\Sigma}_{\phi_{1}^{0} \phi_{2}^{0}}^{(2)}, \\
& \hat{\Sigma}_{h^{0} H^{0}}^{(2)}=\sin \alpha \cos \alpha\left(\hat{\Sigma}_{\phi_{2}^{0} \phi_{2}^{0}}^{(2)}-\hat{\Sigma}_{\phi_{1}^{0} \phi_{1}^{0}}^{(2)}\right)+\cos (2 \alpha) \hat{\Sigma}_{\phi_{1}^{0} \phi_{2}^{0}}^{(2)},
\end{aligned}
$$

where the tree-level propagator matrix is diagonal and $\alpha$ the tree-level mixing angle, see Eqs. (2.2)(2.3). The resulting new contributions to the neutral $C P$-even Higgs-boson self-energies, containing all momentum-dependent and additional constant terms, are assigned to the differences

$$
\Delta \hat{\Sigma}_{a b}^{(2)}\left(p^{2}\right)=\hat{\Sigma}_{a b}^{(2)}\left(p^{2}\right)-\tilde{\Sigma}_{a b}^{(2)}(0), \quad a b=\left\{H^{0} H^{0}, h^{0} H^{0}, h^{0} h^{0}\right\}
$$

Note the tilde (not hat) on $\tilde{\Sigma}^{(2)}(0)$, which signifies that not only the self-energies are evaluated at zero external momentum but also the corresponding counter terms, following Refs. [38-40]. A finite shift $\Delta \hat{\Sigma}^{(2)}(0)$ therefore remains in the limit $p^{2} \rightarrow 0$ due to $\delta m_{A^{0}}^{2(2)}=\operatorname{Re} \Sigma_{A^{0} A^{0}}^{(2)}\left(m_{A^{0}}^{2}\right)$ being computed at $p^{2}=m_{A^{0}}^{2}$ in $\hat{\Sigma}^{(2)}$, but at $p^{2}=0$ in $\tilde{\Sigma}^{(2)}$.

Several checks have been performed on the calculation. Subtracting the finite shift of $\delta m_{A^{0}}^{2(2)}$, the finite shift $\Delta \hat{\Sigma}_{a b}^{(2)}(0)$ in Eq. (2.6) must cancel in the limit of vanishing external momentum. This could be confirmed numerically. Moreover, agreement with previous calculations performed in the zero momentum limit $[38,40]$ was found analytically. All integrals which were deduced analytically from known expressions $[35,41]$ were checked with SECDEC. For more details about the calculational set-up the reader is referred to [30,42].

According to Eq. (2.4), the $C P$-even Higgs-boson masses are determined from the poles of the $h^{0}-H^{0}$-propagator matrix. This is equivalent to solving the equation

$$
\left[p^{2}-m_{h^{0}}^{2}+\hat{\Sigma}_{h^{0} h^{0}}\left(p^{2}\right)\right]\left[p^{2}-m_{H^{0}}^{2}+\hat{\Sigma}_{H^{0} H^{0}}\left(p^{2}\right)\right]-\left[\hat{\Sigma}_{h^{0} H^{0}}\left(p^{2}\right)\right]^{2}=0
$$

yielding the loop-corrected pole masses, $M_{h}$ and $M_{H}$. 


\section{Numerical results}

The following parameter values are adopted for the numerical studies shown below

$$
\begin{aligned}
& m_{t}=173.2 \mathrm{GeV}, M_{\mathrm{SUSY}}=1 \mathrm{TeV}, X_{t}=2 M_{\mathrm{SUSY}}, \mu=200 \mathrm{GeV}, \\
& m_{\tilde{g}}=1500 \mathrm{GeV}, m_{\tilde{t}_{1}}=826.8 \mathrm{GeV}, m_{\tilde{t}_{2}}=1173.2 \mathrm{GeV} .
\end{aligned}
$$

They are oriented at the $m_{h}^{\max }$ scenario described in Ref. [43]. Results for other scenarios and more details can be found in Ref. [30]. In Fig. 2 $\Delta M_{h}$ (left plot) and $\Delta M_{H}$ (right plot) are shown as a function of $m_{A^{0}}$ for $\tan \beta=5$ (blue) and $\tan \beta=20$ (red). In the $m_{h}^{\max }$ scenario for $m_{A^{0}} \gtrsim 200 \mathrm{GeV}$, the additional shift $\Delta M_{h} \sim-60 \mathrm{MeV}$ amounts to the size of the anticipated experimental precision at a linear collider. The contribution to the heavy $C P$-even Higgs-boson mass can reach $-60 \mathrm{MeV}$ for very small or intermediate values of $m_{A^{0}}$, whereas for $m_{A^{0}} \gtrsim 500 \mathrm{GeV}$ a decreasing correction to $M_{H}$ can be observed. The peak in $\Delta M_{H}$ for $\tan \beta=5$ originates from a threshold at $2 m_{t}$.
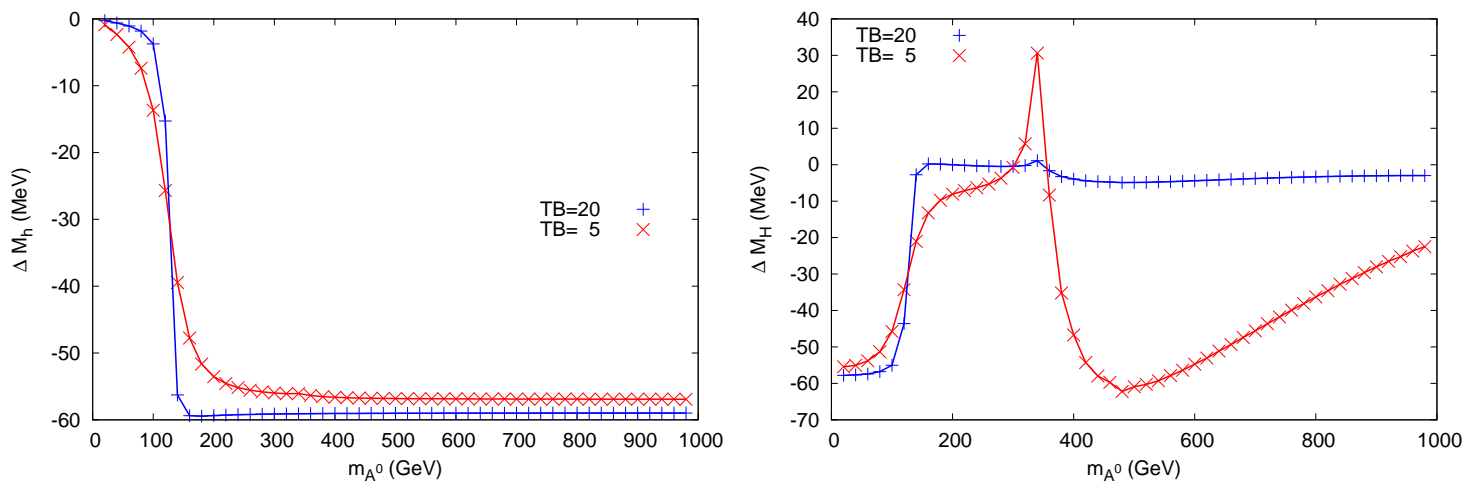

Figure 2: Variation of the mass shifts $\Delta M_{h}, \Delta M_{H}$ with the $A^{0}$-boson mass $m_{A^{0}}$ for $\tan \beta=5$ (blue) and $\tan \beta=20$ (red). The peak in $\Delta M_{H}$ originates from a threshold at $2 m_{t}$.
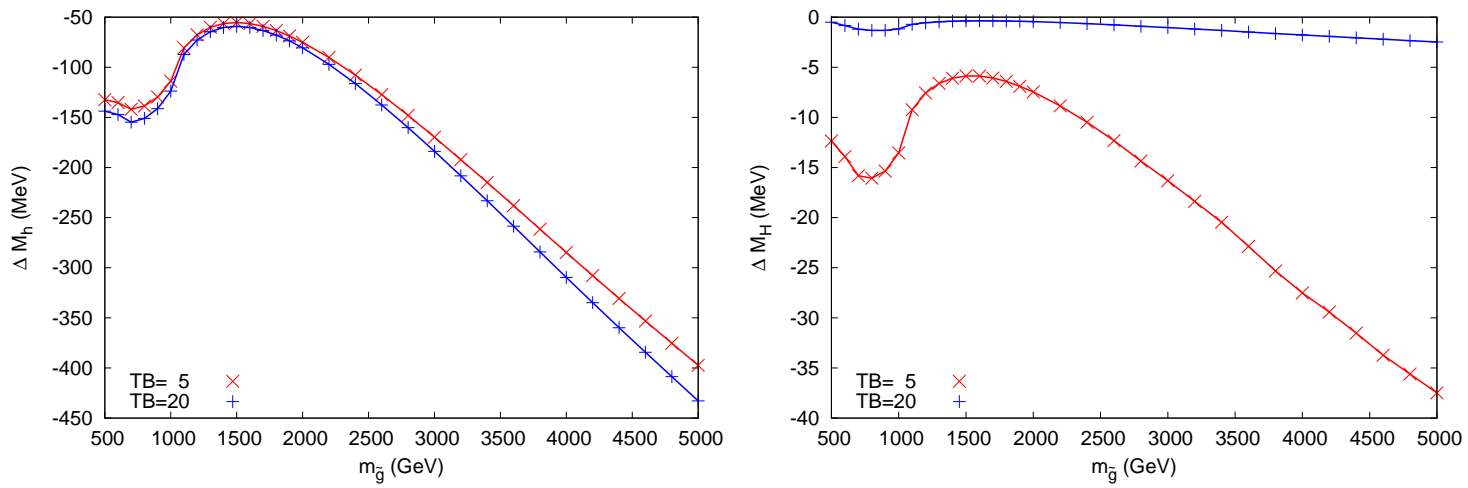

Figure 3: Variation of the mass shifts $\Delta M_{h}, \Delta M_{H}$ with the gluino mass for two different values of $\tan \beta=$ 5,20 and $m_{A^{0}}=250 \mathrm{GeV}$.

Furthermore, the dependence of $M_{h}$ and $M_{H}$ on the gluino mass $m_{\tilde{g}}$ is analyzed in the scenario described above. The results are shown in Fig. B for $\Delta M_{h}$ (left plot) and $\Delta M_{H}$ (right plot) for 
$m_{A^{0}}=250 \mathrm{GeV}$ with the same color coding as in Fig. 2. In the case of $M_{h}$ one can observe that the effects are smallest for $m_{\tilde{g}} \sim 1.5 \mathrm{TeV}$. More sizable shifts occur for larger gluino masses, by more than $-400 \mathrm{MeV}$ for $m_{\tilde{g}} \gtrsim 4 \mathrm{TeV}$, reaching thus the level of the current experimental accuracy in the Higgs-boson mass determination. The corrections to $M_{H}$ do not exceed $-50 \mathrm{MeV}$ in the considered $m_{\tilde{g}}$ range.

\section{Conclusion}

Results for the leading momentum-dependent $\mathscr{O}\left(\alpha_{t} \alpha_{s}\right)$ contributions to the masses of the neutral $C P$-even Higgs-bosons in the MSSM have been presented. They were obtained by calculating the corresponding contributions to the dressed Higgs-boson propagators in the Feynman-

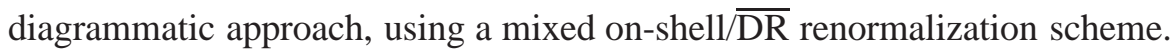

The effect of the new momentum-dependent two-loop corrections on the predictions for the $C P$-even Higgs-boson masses was investigated. The numerical analysis displayed a strong dependence of the light $C P$-even Higgs-boson mass on the value of the gluino mass. For values of $m_{\tilde{g}} \sim 1.5 \mathrm{TeV}$ corrections to $M_{h}$ of about $-50 \mathrm{MeV}$ are found, while for very large gluino masses, $m_{\tilde{g}} \gtrsim 4 \mathrm{TeV}$, the corrections can amount to the level of the current experimental accuracy, i.e. about $500 \mathrm{MeV}$ at the LHC. The effects are mostly below the current and future anticipated experimental accuracies for the heavy $C P$-even Higgs-boson mass. The new results of $\mathscr{O}\left(\alpha_{t} \alpha_{s}\right)$ have been incorporated into the program FEYNHIGGS.

\section{Acknowledgements}

I would like to thank Thomas Hahn, Sven Heinemeyer, Gudrun Heinrich and Wolfgang Hollik for the fruitful collaboration, and Stefano di Vita for comparisons. Furthermore I wish to thank the organizers of Loops and Legs 2014 for the nice and interesting conference.

\section{References}

[1] G. Aad et al. [ATLAS Collaboration], Phys. Lett. B 716 (2012) 1 [arXiv:1207.7214 [hep-ex]].

[2] S. Chatrchyan et al. [CMS Collaboration], Phys. Lett. B 716 (2012) 30 [arXiv:1207.7235 [hep-ex]].

[3] H. Nilles, Phys. Rept. 110 (1984) 1;

H. Haber and G. Kane, Phys. Rept. 117 (1985) 75;

R. Barbieri, Riv. Nuovo Cim. 11 (1988) 1.

[4] J. Ellis, G. Ridolfi and F. Zwirner, Phys. Lett. B 257 (1991) 83;

Y. Okada, M. Yamaguchi and T. Yanagida, Prog. Theor. Phys. 85 (1991) 1;

H. Haber and R. Hempfling, Phys. Rev. Lett. 66 (1991) 1815.

[5] A. Brignole, Phys. Lett. B 281 (1992) 284.

[6] P. Chankowski, S. Pokorski and J. Rosiek, Phys. Lett. B 286 (1992) 307; Nucl. Phys. B 423 (1994) 437 [arXiv:hep-ph/9303309].

[7] A. Dabelstein, Nucl. Phys. B 456 (1995) 25 [arXiv:hep-ph/9503443]; Z. Phys. C 67 (1995) 495 [arXiv:hep-ph/9409375]. 
[8] S. Heinemeyer, W. Hollik and G. Weiglein, Phys. Rev. D 58 (1998) 091701 [arXiv:hep-ph/9803277]; Phys. Lett. B 440 (1998) 296 [arXiv:hep-ph/9807423].

[9] S. Heinemeyer, W. Hollik and G. Weiglein, Eur. Phys. J. C 9 (1999) 343 [arXiv:hep-ph/9812472].

[10] S. Heinemeyer, W. Hollik and G. Weiglein, Phys. Lett. B 455 (1999) 179 [arXiv:hep-ph/9903404].

[11] S. Heinemeyer, W. Hollik, H. Rzehak and G. Weiglein, Eur. Phys. J. C 39 (2005) 465 [arXiv:hep-ph/0411114].

[12] M. Carena, H. Haber, S. Heinemeyer, W. Hollik, C. Wagner, and G. Weiglein, Nucl. Phys. B 580 (2000) 29 [arXiv:hep-ph/0001002].

[13] R. Zhang, Phys. Lett. B 447 (1999) 89 [arXiv:hep-ph/9808299];

J. Espinosa and R. Zhang, JHEP $\mathbf{0 0 0 3}$ (2000) 026 [arXiv:hep-ph/9912236].

[14] G. Degrassi, P. Slavich and F. Zwirner, Nucl. Phys. B 611 (2001) 403 [arXiv:hep-ph/0105096].

[15] R. Hempfling and A. Hoang, Phys. Lett. B 331 (1994) 99 [arXiv:hep-ph/9401219].

[16] A. Brignole, G. Degrassi, P. Slavich and F. Zwirner, Nucl. Phys. B 631 (2002) 195 [arXiv:hep-ph/0112177].

[17] J. Espinosa and R. Zhang, Nucl. Phys. B 586 (2000) 3 [arXiv:hep-ph/0003246].

[18] J. Espinosa and I. Navarro, Nucl. Phys. B 615 (2001) 82 [arXiv:hep-ph/0104047].

[19] A. Brignole, G. Degrassi, P. Slavich and F. Zwirner, Nucl. Phys. B 643 (2002) 79 [arXiv:hep-ph/0206101].

[20] G. Degrassi, A. Dedes and P. Slavich, Nucl. Phys. B 672 (2003) 144 [arXiv:hep-ph/0305127].

[21] M. Carena, J. Espinosa, M. Quirós and C. Wagner, Phys. Lett. B 355 (1995) 209 [arXiv:hep-ph/9504316];

M. Carena, M. Quirós and C. Wagner, Nucl. Phys. B 461 (1996) 407 [arXiv:hep-ph/9508343].

[22] J. Casas, J. Espinosa, M. Quirós and A. Riotto, Nucl. Phys. B 436 (1995) 3, [Erratum-ibid. B 439 (1995) 466] [arXiv:hep-ph/9407389].

[23] S. Heinemeyer, W. Hollik and G. Weiglein, Comput. Phys. Commun. 124 (2000) 76 [arXiv:hep-ph/9812320];

T. Hahn, S. Heinemeyer, W. Hollik, H. Rzehak and G. Weiglein, Comput. Phys. Commun. 180 (2009) 1426; see: www. feynhiggs.de .

[24] S. Martin, Phys. Rev. D 71 (2005) 016012 [arXiv:hep-ph/0405022].

[25] S. Martin, Phys. Rev. D 65 (2002) 116003 [arXiv:hep-ph/0111209]; Phys. Rev. D 66 (2002) 096001 [arXiv:hep-ph/0206136]; Phys. Rev. D 67 (2003) 095012 [arXiv:hep-ph/0211366]; Phys. Rev. D 68 (2003) 075002 [arXiv:hep-ph/0307101]; Phys. Rev. D 70 (2004) 016005 [arXiv:hep-ph/0312092]; Phys. Rev. D 71 (2005) 116004 [arXiv:hep-ph/0502168]; Phys. Rev. D 75 (2007) 055005 [arXiv:hep-ph/0701051]; S. Martin and D. Robertson, Comput. Phys. Commun. 174 (2006) 133 [arXiv:hep-ph/0501132].

[26] R. Harlander, P. Kant, L. Mihaila and M. Steinhauser, Phys. Rev. Lett. 100 (2008) 191602 [Phys. Rev. Lett. 101 (2008) 039901] [arXiv:0803.0672 [hep-ph]]; JHEP 1008 (2010) 104 [arXiv:1005.5709 [hep-ph]].

[27] T. Hahn, S. Heinemeyer, W. Hollik, H. Rzehak and G. Weiglein, Phys. Rev. Lett. 112 (2014) 141801, arXiv:1312.4937 [hep-ph]. 
[28] G. Degrassi, S. Heinemeyer, W. Hollik, P. Slavich and G. Weiglein, Eur. Phys. J. C 28 (2003) 133 [arXiv:hep-ph/0212020].

[29] M. Frank, T. Hahn, S. Heinemeyer, W. Hollik, H. Rzehak and G. Weiglein, JHEP 0702 (2007) 047 [arXiv:hep-ph/0611326].

[30] S. Borowka, T. Hahn, S. Heinemeyer, G. Heinrich and W. Hollik, Eur. Phys. J. C 74 (2014) 2994 arXiv:1404.7074 [hep-ph].

[31] J. Küblbeck, M. Böhm and A. Denner, Comput. Phys. Commun. 60 (1990) 165;

T. Hahn, Comput. Phys. Commun. 140 (2001) 418 [arXiv:hep-ph/0012260];

T. Hahn and C. Schappacher, Comput. Phys. Commun. 143 (2002) 54 [arXiv:hep-ph/0105349]. The program and the user's guide are available via www. feynarts. de .

[32] T. Fritzsche, T. Hahn, S. Heinemeyer, F. von der Pahlen, H. Rzehak and C. Schappacher, Comput. Phys. Commun. 185 (2014) 1529, arXiv:1309.1692 [hep-ph].

[33] T. Hahn and M. Pérez-Victoria, Comput. Phys. Commun. 118 (1999) 153 [arXiv:hep-ph/9807565].

[34] G. Weiglein, R. Scharf and M. Böhm, Nucl. Phys. B 416 (1994) 606 [arXiv:hep-ph/9310358]; G. Weiglein, R. Mertig, R. Scharf and M. Böhm, in New Computing Techniques in Physics Research 2, ed. D. Perret-Gallix (World Scientific, Singapore, 1992), p. 617.

[35] A. I. Davydychev and J. B. Tausk, Nucl. Phys. B 397 (1993) 123.

[36] S. Borowka, J. Carter and G. Heinrich, Comput. Phys. Commun. 184 (2013) 396 [arXiv:1204.4152 [hep-ph]].

[37] S. Borowka and G. Heinrich, Comput. Phys. Commun. 184 (2013) 2552 [arXiv:1303.1157 [hep-ph]].

[38] S. Heinemeyer, W. Hollik and G. Weiglein, Phys. Rev. D 58 (1998) 091701 [hep-ph/9803277].

[39] S. Heinemeyer, W. Hollik and G. Weiglein, Phys. Lett. B 440 (1998) 296 [hep-ph/9807423].

[40] S. Heinemeyer, W. Hollik and G. Weiglein, Eur. Phys. J. C 9 (1999) 343 [hep-ph/9812472].

[41] F. A. Berends, A. I. Davydychev, V. A. Smirnov and J. B. Tausk, Nucl. Phys. B 439 (1995) 536 [hep-ph/9410232].

[42] S. Borowka and G. Heinrich, PoS RADCOR 2013 (2014) 009 [arXiv:1311.6476 [hep-ph]].

[43] M. Carena, S. Heinemeyer, O. Stål, C. Wagner and G. Weiglein, Eur. Phys. J. C 73 (2013) 2552 [arXiv:1302.7033 [hep-ph]]. 